\title{
The fate of murine double minute $X(M d m X)$ is dictated by distinct signaling pathways through murine double minute 2 (Mdm2)
}

\author{
Paula M. Hauck ${ }^{1}$, Eric R. Wolf ${ }^{2}$, David J. Olivos III $^{1}$, Ciaran P. McAtarsney ${ }^{1}$ and \\ Lindsey D. Mayo ${ }^{1,2,3}$ \\ ${ }^{1}$ Department of Pediatrics, Herman B Wells Center for Pediatrics Research, Indianapolis, Indiana, 46202, United States of America \\ ${ }^{2}$ Department of Biochemistry and Molecular Biology, Indiana University School of Medicine, Indianapolis, Indiana, 46202, \\ United States of America \\ ${ }^{3}$ Indiana University Simon Cancer Center, Indiana University School of Medicine, Indianapolis, Indiana, 46202, United States \\ of America
}

Correspondence to: Lindsey D. Mayo, email: Idmayo@iu.edu

Keywords: MdmX; Mdm2; neddylation; MLN4924; ATM

Received: July 19, $2017 \quad$ Accepted: October 05, $2017 \quad$ Published: November 06, 2017

Copyright: Hauck et al. This is an open-access article distributed under the terms of the Creative Commons Attribution License 3.0 (CC BY 3.0), which permits unrestricted use, distribution, and reproduction in any medium, provided the original author and source are credited.

\section{ABSTRACT}

Mouse double minute 2 (Mdm2) and MdmX dimerize in response to low levels of genotoxic stress to function in a ubiquitinating complex, which signals for destabilization of p53. Under growth conditions, Mdm2 functions as a neddylating ligase, but the importance and extent of $\mathrm{MdmX}$ involvement in this process are largely unknown. Here we show that when Mdm2 functions as a neddylating enzyme, MdmX is stabilized. Furthermore, we demonstrate that under growth conditions, MdmX enhances the neddylation activity of Mdm2 on p53 and is a substrate for neddylation itself. Importantly, MdmX knockdown in MCF-7 breast cancer cells resulted in diminished neddylated p53, suggesting that MdmX is important for Mdm2-mediated neddylation. Supporting this finding, the lack of $\mathrm{MdmX}$ in transient assays or in p53/MdmX-/- MEFs results in decreased or altered neddylation of p53 respectively; therefore, MdmX is a critical component of the Mdm2-mediated neddylating complex. c-Src is the upstream activator of this Mdm2-MdmX neddylating pathway and loss of Src signaling leads to the destabilization of MdmX that is dependent on the RING (Really Interesting New Gene) domain of MdmX. Treatment with a small molecule inhibitor of neddylation, MLN4924, results in the activation of Ataxia Telangiectasia Mutated (ATM). ATM phosphorylates Mdm2, converting Mdm2 to a ubiquitinating enzyme which leads to the destabilization of MdmX. These data show how distinct signaling pathways engage neddylating or ubiquitinating activities and impact the Mdm2-MdmX axis.

\section{INTRODUCTION}

Post-translational modifications such as ubiquitin, sumo, nedd8, and ISG15, are essential for a myriad of fundamental processes in the cell. These modifications can induce conformational changes to recruit binding partners, prevent or enhance interactions, or result in changes in cellular localization. E1, E2 and E3 enzymes are responsible for conjugating these covalent modifications to proteins. Mouse double minute $2(\mathrm{Mdm} 2)$ is one of over a thousand E3 ubiquitin ligases. Mdm2 is an oncoprotein that is elevated in $10 \%$ of all human cancers and $40-80 \%$ of high-grade human tumors $[1,2]$. It is most widely recognized for its role in facilitating ubiquitination of $\mathrm{p} 53$, which can lead to p53 destabilization under conditions of genotoxic stress. 
In response to low levels of DNA damage, Mdm2 has been described to act: as a monomer after phosphorylation by Ataxia Telangiectasia Mutated (ATM) $[3,4]$, as a homodimer [4-10], or as a heterodimer with MdmX [5-9, 11-15] after phosphorylation by c-Abl [16] in a ubiquitination complex. The post-translational modifications that dictate whether Mdm2 acts as a monomer, homo- or heterodimer are only beginning to be elucidated. While the Mdm2-MdmX heterodimer has been shown to have ubiquitin ligase activity [11], it is most commonly accepted that MdmX does not have ubiquitin ligase activity by itself. Most studies have investigated MdmX-Mdm2-p53 signaling under DNA damage, and agree that $\mathrm{MdmX}$ is destabilized under these conditions due to Mdm2-mediated ubiquitination of MdmX (reviewed by [17]). MdmX is related to Mdm2 (C-terminal domains are $46 \%$ identical), and can bind directly to the transactivation domain of $\mathrm{p} 53$ to inhibit p53 transcriptional activity [18]. MdmX is also considered an oncogene by its ability to transform primary cells and is amplified in approximately $65-85 \%$ of infiltrating ductal breast carcinomas [19], retinoblastomas [20] and metastatic melanomas [21].

Interestingly, $\mathrm{Mdm} 2$ is one of a small number of E3 ligases that can mediate neddylation [22]. We previously demonstrated that under growth conditions, Mdm2 is a substrate for Src phosphorylation at Y281 and $\mathrm{Y} 302$, which converts $\mathrm{Mdm} 2$ from a ubiquitin to a Nedd8 E3 ligase [23]. This Nedd8 activity of Mdm2 results in neddylated p53 that has increased stability but is transcriptionally inactive.

In the current study, we show that conversion of Mdm2 to a neddylating enzyme by growth signaling results in Mdm2 forming a heterodimer with MdmX. This dimerization is dependent on Src phosphorylation of Mdm2. We also show that MdmX is conjugated with Nedd8. As expected, in the presence of Src, Mdm2, and MdmX, p53 neddylation is enhanced, thereby blocking p53 transcriptional activity. However, inhibition of the E1 neddylating enzyme by MLN4924 (Pevonedistat -Takeda /Millennium Pharmaceuticals, Inc.) leads to the activation of $\mathrm{p} 53$. This is a multi-faceted mechanism whereby MdmX levels are dramatically decreased in addition to the prevention of neddylation. This destabilization is dependent on Mdm2. Our data show how multiple signaling pathways regulate the stability of MdmX and the regulation of $\mathrm{p} 53$.

\section{RESULTS}

\section{Modulation of MdmX levels in response to post- translational modifications}

$\mathrm{Mdm} 2$ acts as a neddylating ligase in response to $\mathrm{Src}$ phosphorylation [23], but the effect on MdmX is unknown. When MdmX was transiently co-expressed with Mdm2 in H1299 cells, MdmX levels decreased dramatically compared to MdmX expression alone (Figure 1A top panel). This result was expected as down-regulation of $\mathrm{MdmX}$ in response to overexpression of Mdm2 has been reported in the literature $[24,25]$. This affect was reversed with the addition of constitutively active Src (CA-Src). To show that these observations were dependent on the Src phosphorylation sites on Mdm2, we used the Src phosphorylation double mutant Y281/302F Mdm2 in the presence of CA-Src and MdmX. We found that CA-Src failed to rescue MdmX levels in the presence of the Mdm2 Y281/302F mutant (Figure 1A bottom panel). These data provide evidence that Src phosphorylation of $\mathrm{Mdm} 2$ prevents Mdm2-mediated destabilization of MdmX.

We next examined if we could see similar results in an endogenous system through pharmacological approaches. Serum starved MCF7 cells were treated with DMSO and EGF (epidermal growth factor), or PP1 (an inhibitor of Src) and EGF. Mdm2 and MdmX increased in the presence of EGF (Figure 1B). This increase in MdmX levels was attenuated in the presence of PP1. While PP1 blocks c-Src activity, Src abundance was not affected under these conditions (Figure 1B). These endogenous results show that MdmX levels are sensitive to the $\mathrm{Src} /$ Mdm2 pathway.

Compared to $\mathrm{Mdm} 2$ and $\mathrm{p} 53$, there is much less known about protein interactions that affect MdmX levels and function. When Y99 of MdmX is phosphorylated by c-Abl, interaction with p53 is prevented [26]. In addition, c-Abl drives Mdm2-MdmX complex formation, which leads to degradation of this complex [16]. Mutation of P369G disrupts binding to 14-3-3, which normally occurs upon irradiation exposure to UV to inhibit p53 ubiquitination [27]. We tested if a Y99F and a P369G mutant of MdmX were altered in response to Src signaling by transient expression in H1299 cells. Similar to the results shown in Figure 1A, the levels of MdmX, as well as each of the mutants, increased in the presence of CASrc (Figure 1C). Overall, these results show that growth signaling results in increased protein levels of MdmX.

\section{Mdm2 and MdmX interact under growth conditions}

Since MdmX levels were dependent on Mdm2, we wanted to determine where MdmX and Mdm2 were localized in the cell under growth conditions (+EGF). Using immunofluorescence confocal microscopy we show that $\mathrm{MdmX}$ and $\mathrm{Mdm} 2$ were predominantly co-localized in the nucleus, but also in the cytoplasm of MCF7 cells (Figure 2A).

We then investigated if MdmX would co-purify with $\mathrm{Mdm} 2$. Indeed, in response to active Src, Mdm2 co-purified with MdmX when transiently expressed in H1299 cells (Figure 2B). In addition, every MdmX mutant tested was able to co-purify with $\mathrm{Mdm} 2$ in the presence 


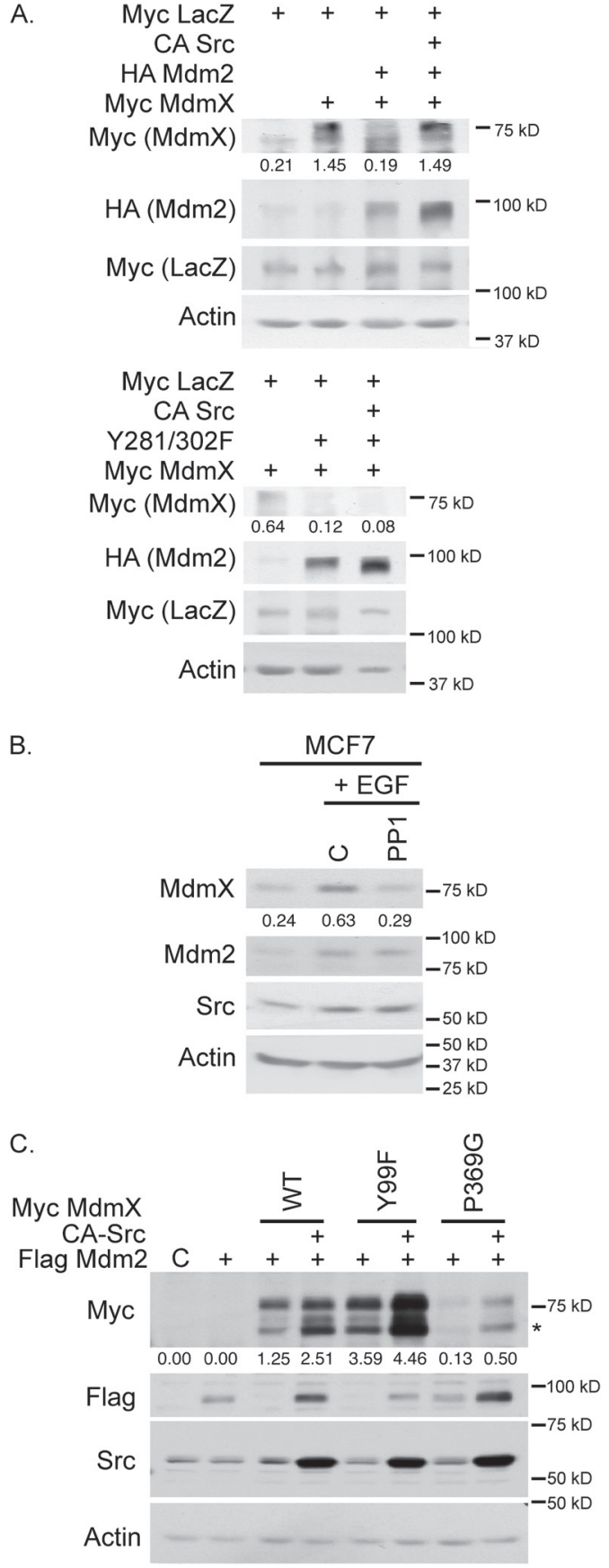

Figure 1: Conversion of Mdm2 to a neddylating ligase results in increased levels of $\mathrm{MdmX}$ as detected by western blot analysis. (A) Without CA-Src in the H1299 transient transfections, Mdm2 acts as a ubiquitinating ligase and $\mathrm{MdmX}$ levels are decreased. The addition of CA-Src results in recovery of MdmX levels (top). The Y281-302F (Src phosphorylation sites) Mdm2 mutant cannot act as a neddylating ligase and MdmX levels cannot be rescued by CA-Src (bottom). (B) Endogenous levels of MdmX and Mdm2 increase with EGF treatment and this increase is attenuated with PP1 treatment. (C) Similar transfection as in A, but with various MdmX mutants showing that the CA-Src/Mdm2 mediated increase in $\mathrm{MdmX}$ levels is independent of these mutations. Asterisks indicate nonspecific bands. All panels: number underneath the lanes represent the ratio of the intensity of the MdmX band to the intensity of the actin band as determined by ImageJ. of CA-Src (Figure 2B). We observed that the amount of $\mathrm{Mdm} 2$ immunoprecipitated in the presence of Src was increased in our experiments. To ensure that the increase in interaction between $\mathrm{MdmX}$ and Mdm2 was due to posttranslational modifications and not due solely to increased expression levels, we used a cell free approach with bacterially produced recombinant proteins and found that the interaction between GST- Mdm2 and His-MdmX was increased in the presence of active Src (Figure 2C). This finding suggests that binding of $\mathrm{Mdm} 2$ with $\mathrm{MdmX}$ is not an artifact of increased protein levels and the increase in association was in response to phosphorylation.

To validate these findings, the endogenous interaction between $\mathrm{MdmX}$ and $\mathrm{Mdm} 2$ under growth conditions was investigated. MdmX co-purified with $\mathrm{Mdm} 2$ that was immunoprecipitated from EGF treated MCF7 cells (Figure 2D). Taken together, these results demonstrate that under growth conditions, when Mdm2 is phosphorylated by Src and acting as a neddylating enzyme, Mdm2 interacts with and increases MdmX.

The complex formation and increased MdmX abundance could be a result of neddylation by Mdm2. To test this, we transiently transfected H1299 cells with combinations of His-Nedd8, Myc-MdmX, HA-Mdm2 and CA-Src and performed a nickel pull-down assay. We found that $\mathrm{MdmX}$ was neddylated only in the presence of both Mdm2 and CA-Src (Figure 2E). This result also shows that in the presence of active Src, MdmX was unable to be neddylated in the absence of Mdm2 (Figure 2E). Reprobing the membrane showed that neddylation of Mdm2 also depends on Src (Supplementary Figure 1). Taken together, our data demonstrates that Mdm2 and $\mathrm{MdmX}$ form a neddylating complex that leads to neddylation of MdmX.

\section{MdmX enhances neddylation of p53}

Considering that $\mathrm{Mdm} 2$ and $\mathrm{MdmX}$ form an active neddylating complex, we next examined if this complex was able to neddylate p53. A nickel pull-down assay showed that neddylation of $\mathrm{p} 53$ was more robust in the presence CA-Src and MdmX (Figure 3A and 3B). In the absence of exogenous MdmX, p53 neddylation was noticeably decreased. As predicted, transfection of kinase dead Src $(\mathrm{KD}-\mathrm{Src})$ resulted in less p53 neddylation compared to no Src overexpression (Figure 3A and 3B). To provide additional support for the requirement of an Mdm2-MdmX neddylation complex, we repeated the nickel pull-down assay and included MLN4924. Treatment with MLN4924 diminished neddylation of p53 despite expression of Mdm2, MdmX, and CA-Src (Figure 3B). Next, we transiently transfected mutant p53 D281G (to prevent lethality caused by p53 expression), CA-Src, and His-Nedd8 into p53-/-, p53 -/- MdmX -/-, p53-/Mdm2-/-, and p53-/- MdmX-/- Mdm2 -/- MEFs. A nickel pull-down was then conducted, and while the presence of Mdm2 alone was sufficient for the faster migrating 
forms of neddylated p53, MdmX was necessary for the slower migrating forms of neddylated p53 (Figure 3C) in a murine system.

To further our analysis, we immunoprecipitated endogenous Nedd 8 from shControl and shMdmX MCF7 cells and blotted for p53. We found that neddylated p53 was observed in shControl cells, but not in the shMdmX cells (Figure 3D; total p53 levels shown in Supplementary Figure 2). This endogenous data provides insight into signaling and neddylation of p53 under physiological conditions. Collectively, our data supports a key role for MdmX in enhancing Mdm2-mediated neddylation of p53.

\section{MLN4924 treatment results in decreased levels of MdmX}

Since MdmX is neddylated (Figure 2E) and is involved in neddylating p53 (Figure 3), we wanted to investigate the effect of inhibiting neddylation with MLN4924, a small molecule inhibitor of Nedd8activating enzyme. As expected, incubation of MCF7 and U87 cells with $0.3 \mu \mathrm{m}$ MLN4924 resulted in decreased protein levels of MdmX (Figure 4A). Furthermore, this decrease was abrogated with treatment of $20 \mu \mathrm{M}$ MG132, which suggests that MLN4924 results in proteosomal degradation of $\mathrm{MdmX}$.

Since Mdm2 can ubiquitinate $\mathrm{MdmX}$ and target it for degradation through the proteosome, we examined whether Mdm2 was necessary for this MLN4924-mediated decrease of MdmX. Considering that genetically removing Mdm2 from cells that are wild type for p53 is lethal, we knocked out Mdm2 using CRISPR/Cas9 targeted to Exon 12 of Mdm2 in TMD231 cells [a derivative of MDA231 (mutant p53 R280K)]. After confirmation of $\mathrm{Mdm} 2$ knockout (Figure 4B left panel), cells were treated with MLN4924. As expected, a decrease in MdmX levels was detected in the vector control cells, which was not evident in the Mdm2 knock-out cells (Figure 4B right panel). This result verifies that $\mathrm{MdmX}$ degradation in response to MLN4924 is dependent on Mdm2.

To further explore this mechanism of Mdm2mediated MdmX destabilization in response to MLN4924, we generated several MCF7 and U87 cell lines by viral transduction: vector control, wildtype MdmX, or MdmX with a C-terminal truncation that lacks a RING domain ( $\triangle \mathrm{C} \mathrm{MdmX)}$ and is unable to interact with Mdm2 [14]. Figure 4C shows that, similar to Figure 4A, MLN4924 treatment decreased wildtype MdmX levels. However, this drug had no effect on $\triangle \mathrm{C} \mathrm{MdmX}$, suggesting that binding with Mdm2 (via the RING domain) is necessary for MLN4924 mediated decrease of $\mathrm{MdmX}$.

The Mdm2-mediated decrease of MdmX could be due solely to the inhibition of neddylation by MLN4924. Alternatively, we postulated that MLN4924 could be converting $\mathrm{Mdm} 2$ to be a ubiquitinating enzyme as
A.
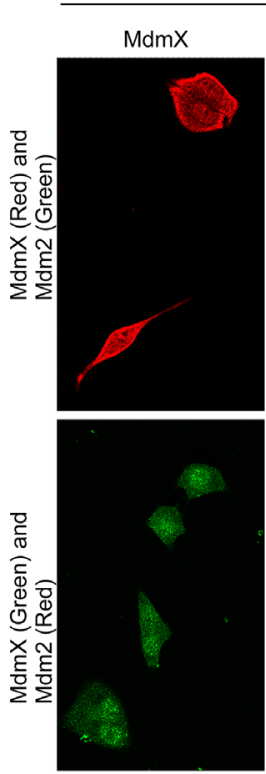

MCF7
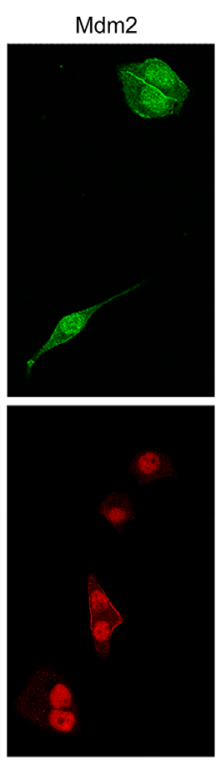

B.
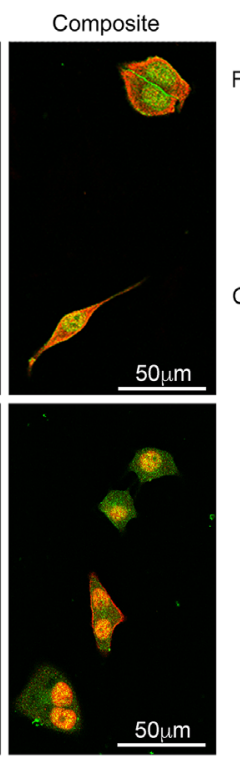
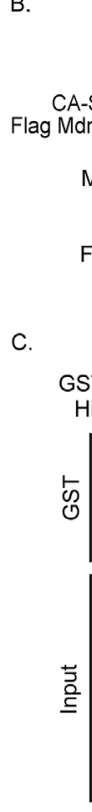

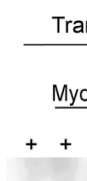

Transfection in H1299 IP: Flag $\operatorname{Myc} X$ MycX yc X Y99F P369G
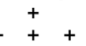

$++$

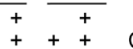

D.
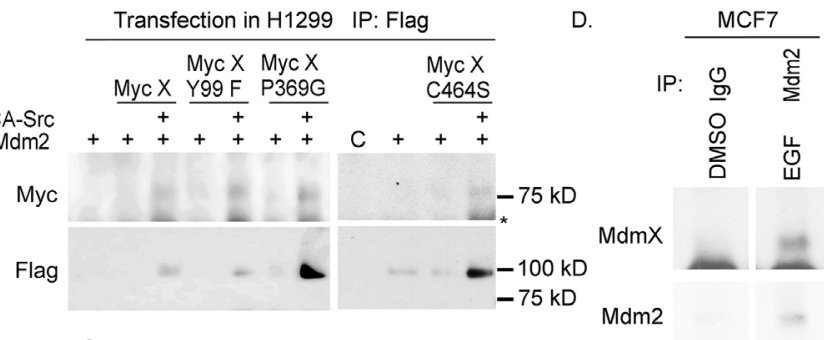

E.

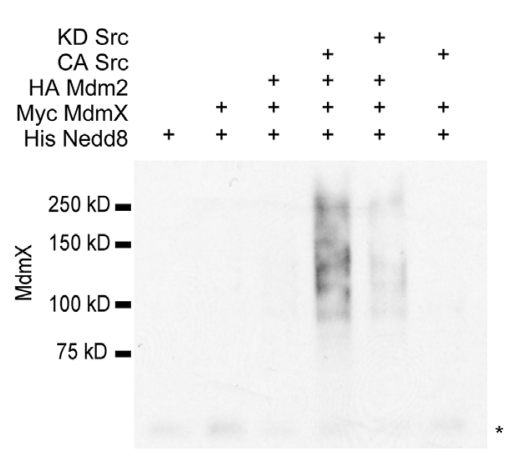

Figure 2: MdmX and Mdm2 interact under growth conditions. (A) Confocal microscopy shows that MdmX and Mdm2 (red and green respectively in the top row or green and red respectively in the bottom row) are co-localized in the nucleus. (B-D) MdmX is in a complex with Mdm2 as determined from western blot analysis of immunoprecipitated extracts from transiently transfected H1299 cells (B), recombinant GST-Pulldowns (C), and immunoprecipitated MCF7 whole cell lysates (lanes spliced from the same gel after formatting) (D). (E) Western blot of a nickel pull-down of His-tagged Nedd8 showing that MdmX is neddylated in a CA-Src and Mdm2 dependent fashion. Asterisks indicate non-specific bands. 
MLN4924 was previously reported to activate ATM [28]. ATM has been shown to phosphorylate Mdm2 [3, 4, 29, 30] and MdmX [31] resulting in MdmX degradation after genotoxic stress. To determine if ATM was involved in MLN4924-mediated degradation of MdmX, we treated MCF7 cells with a combination of MLN4924 and caffeine (an ATM inhibitor). Inhibiting ATM rescued the MLN4924-mediated decreased in wildtype MdmX levels (Figure 4C). This result was validated with a more specific ATM inhibitor (KU-55933) (Supplementary Figure 3). Taken together, these results suggest that MLN4924 activates ATM, which then results in Mdm2mediated degradation of $\mathrm{MdmX}$ through the ring domain of $\mathrm{MdmX}$.

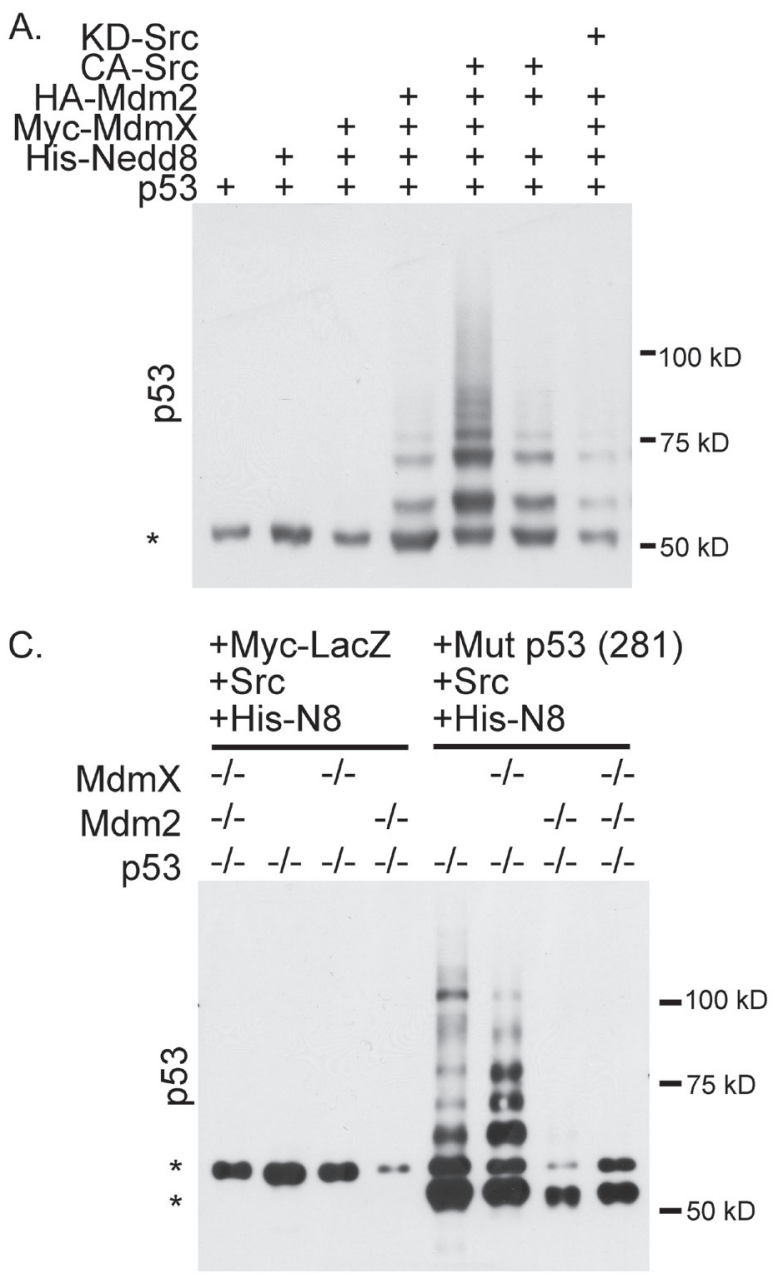

\section{p53 is phosphorylated, stabilized and activated by the MLN4924-ATM-Mdm2-MdmX pathway}

As p53 is a substrate for ATM, we wanted to confirm that ATM was activated by MLN4924. Upon MLN4924 treatment, pS15 of p53 (an established ATM phosphorylation site) was detected, thus indicating ATM activation (Figure 5A). This phosphorylation was attenuated with the addition of caffeine, supporting that ATM was the responsible kinase. In addition, the amount of total p53 increased with MLN4924 treatment and this result was mitigated with the combination of MLN4924 and caffeine (Figure 5A).

Previously, we have shown that increased p53 levels do not necessarily result in increased activity. To assess

B.

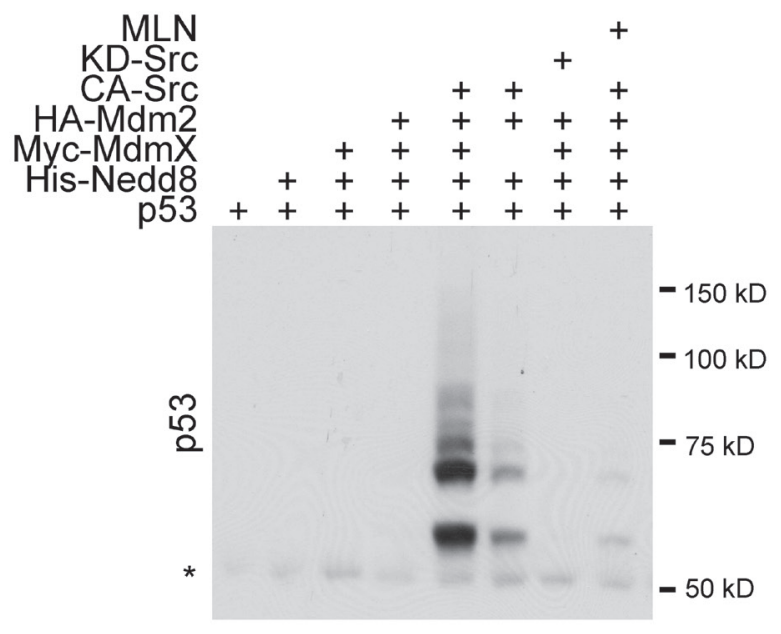

D.

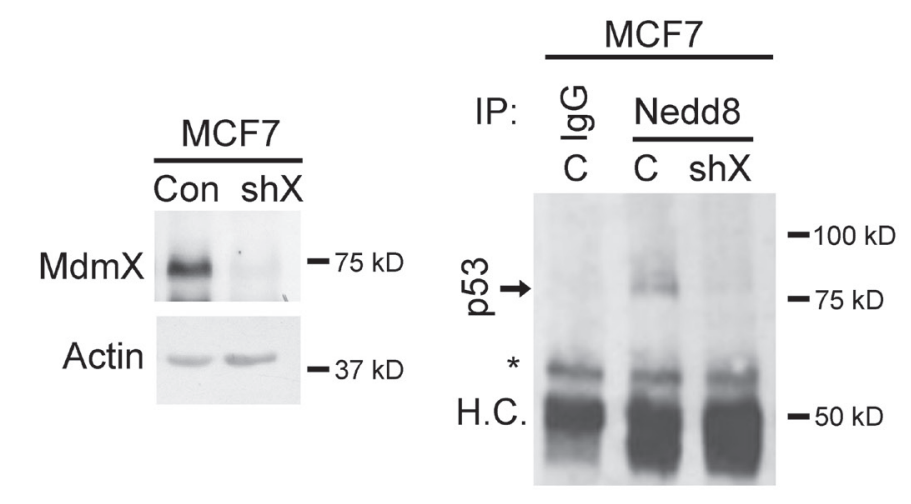

Figure 3: Wildtype and mutant p53 is neddylated in a MdmX-dependent manner as determined by western blot analysis. (A) Nickel pull-down of His-Nedd8 showing that neddylation of p53 is dependent on MdmX and CA-Src. (B) Same Nickel pull-down as in A, but with the addition of MLN4924. MLN4924 decreases neddylation of p53. (C) Transient transfection of MEFs with CA-Src. His-Nedd8 and either Myc-LacZ or Mutp53. The presence of MdmX changes the banding pattern of neddylated p53. (D) MdmX was silenced in shMdmX MCF7 cells (left panel). Neddylated p53 was immunoprecipitated in the presence of MdmX, but not when MdmX was knocked down (right panel) (H.C.= Heavy Chain). All Panels: Asterisks indicate non-specific bands. 
A.

MCF7
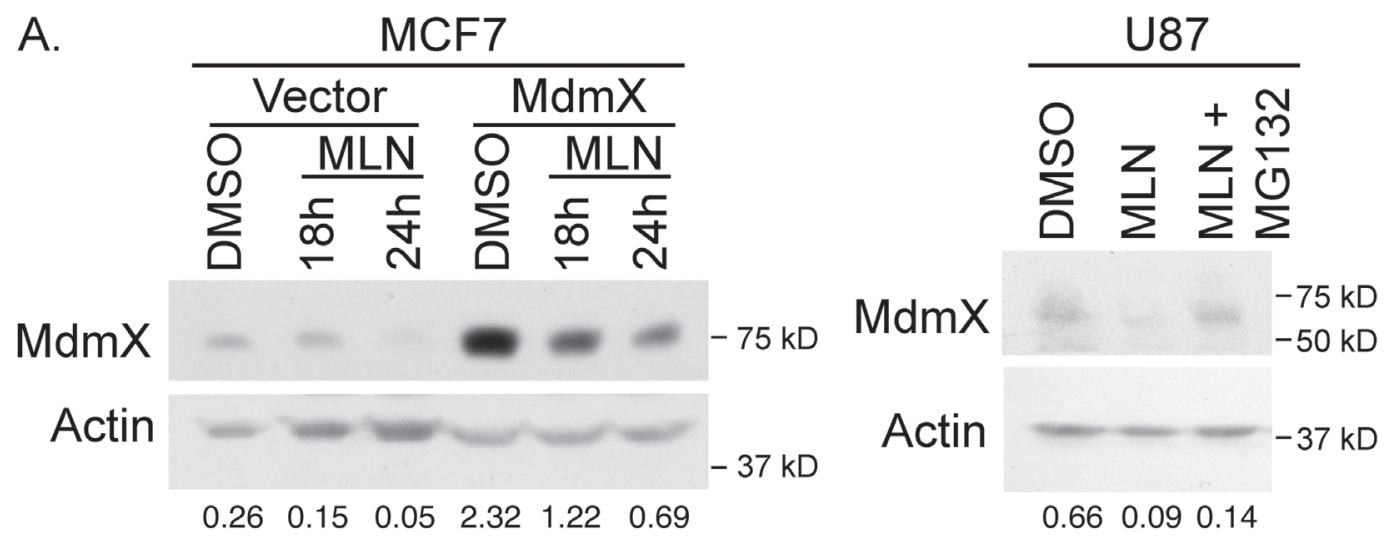

B.

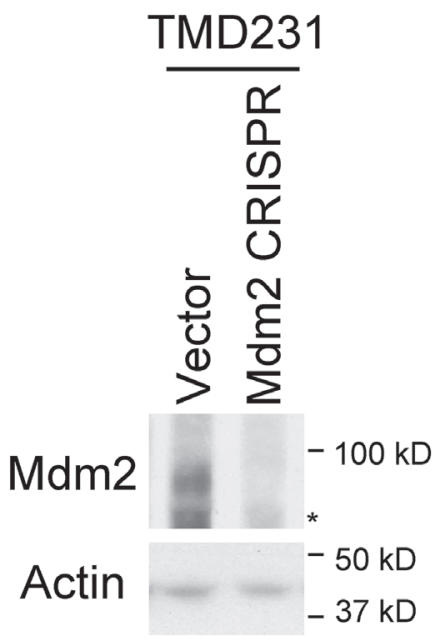

TMD231

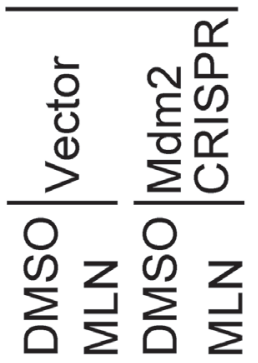

$-75 \mathrm{kD}$

$\operatorname{MdmX}$

$-50 \mathrm{kD}$

Actin $=-2-50 \mathrm{kD}$

C.

MCF7

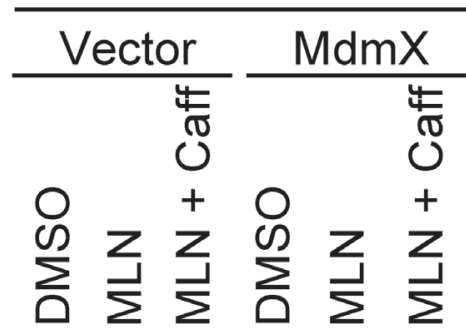

$-150 \mathrm{kD}$

$-100 k D$
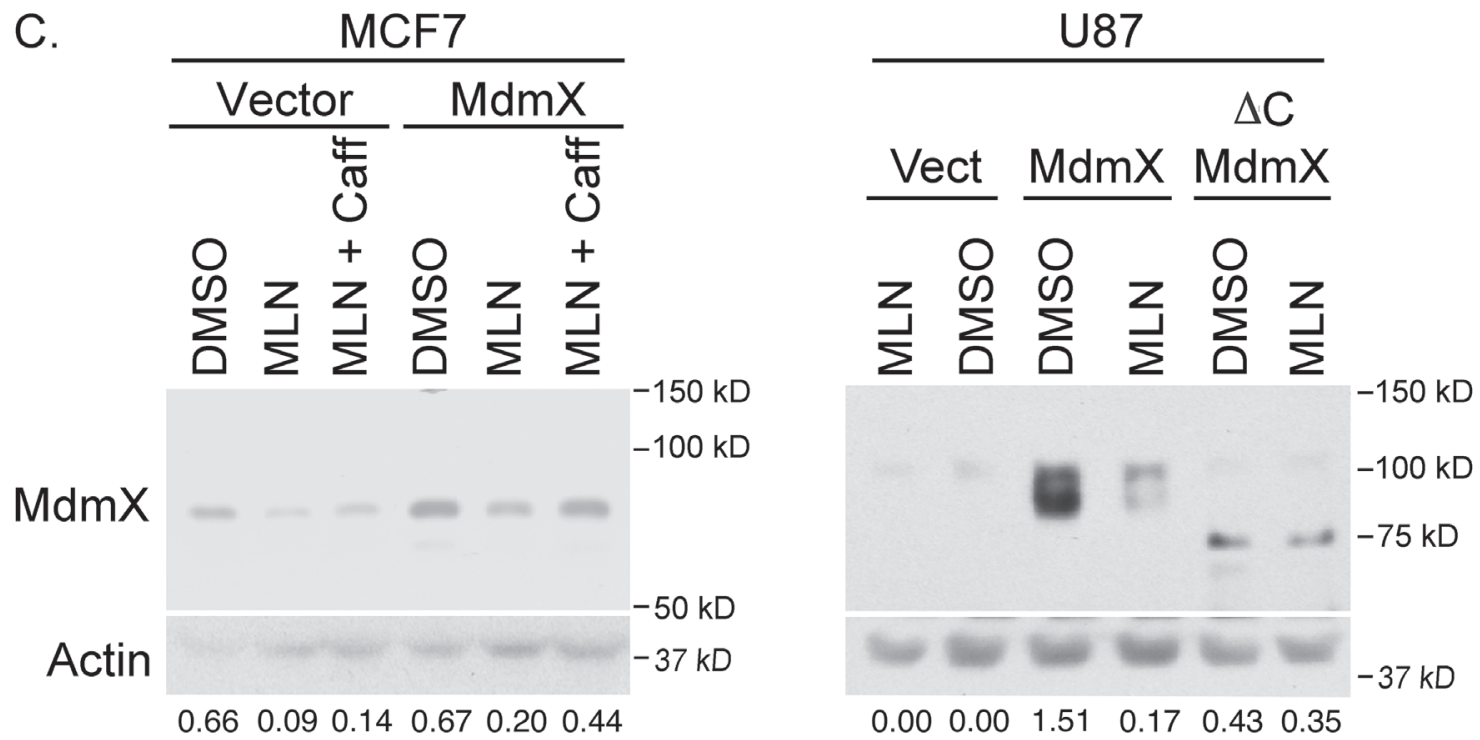

Figure 4: Westerns showing that MLN4924 decreases MdmX levels and this decrease depends on the proteasome, Mdm2, the C-terminus of MdmX and ATM activation. (A) MLN4924 treatment decreases MdmX levels in both MCF7 (left panel) and U87 (right panel). Concurrent treatment with $20 \mu \mathrm{M}$ MG132 (Velcade) rescues MdmX levels. (B) CRISPRed TMD231 cells that lack full length Mdm2 (left panel) do not have decreased MdmX in response to MLN4924 (right panel). (C) Co-treatment with 2 mM caffeine (ATM inhibitor) prevents the MLN4924-mediated decrease in MdmX levels. Therefore, intact signaling through the ATM pathway is required. Also, MdmX lacking the C-terminus is unresponsive to MLN4924. Panels A and C: number underneath the lanes represent the ratio of the intensity of the MdmX band to the intensity of the actin band as determined by ImageJ. 
p53 activity, we investigated the effect of MLN4924 treatment on p21 and found that p21 was also increased with MLN4924 treatment (Figure 5B). This result suggests that p53 is transcriptionally active upon MLN4924 treatment.
Lastly, we conducted fluorescence-activated cell sorting to determine if there were any aberrations in the cell cycle. Treatment of actively growing MCF7 cells with MLN4924 for $18 \mathrm{~h}$ resulted in a higher proportion of cells in the G1 phase and less cells in the G2 phase of

A.

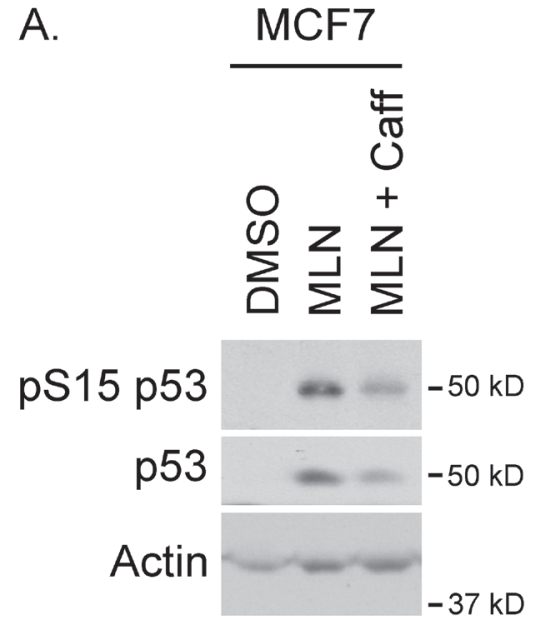

U87

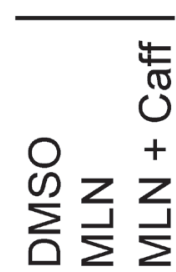

B.
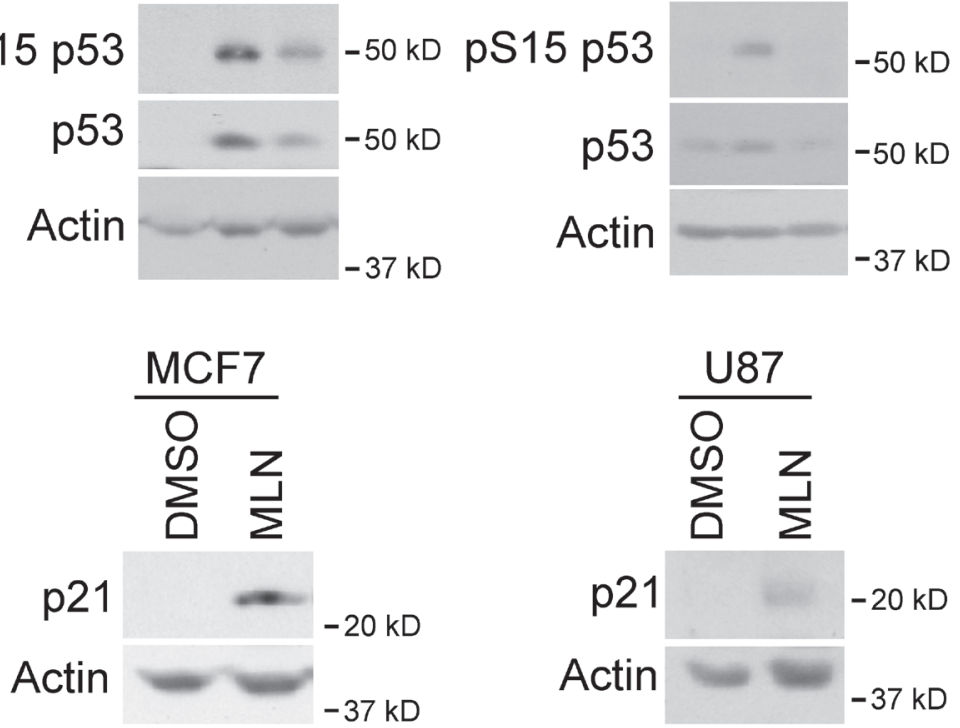

C.

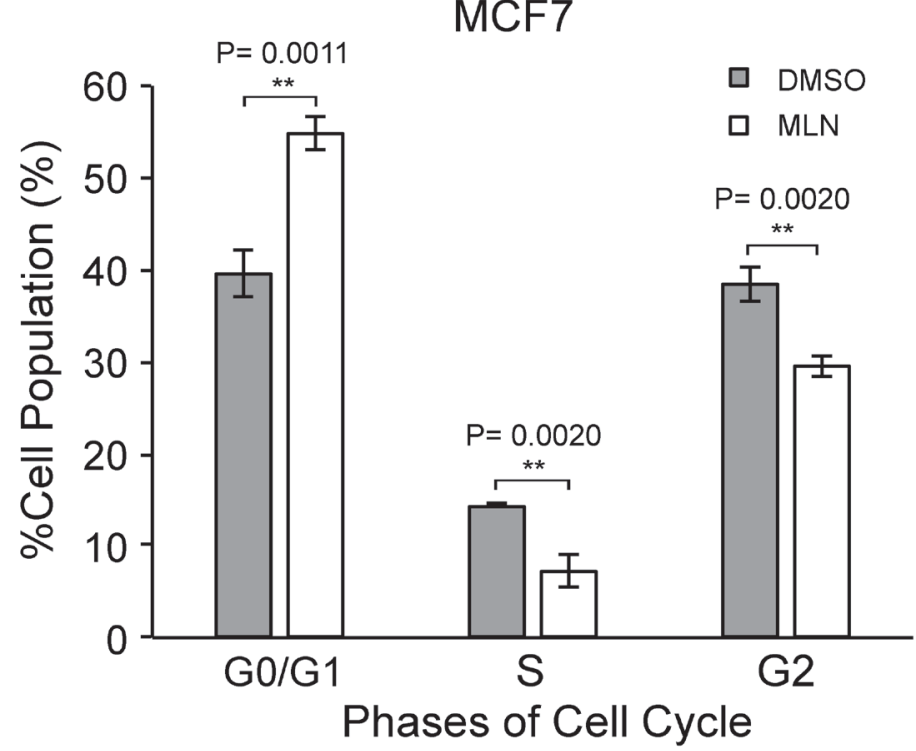

Figure 5: MLN4924 results in p53 activation and cell cycle arrest. (A) Western blots of MCF7 (left) and U87 (right) extracts showing that S15 of p53 is phosphorylated in response to MLN4924 treatment but not with the addition of caffeine. (B) Western analysis of extracts from MCF7 (left) and U87 (right) cells showing increased levels of p21 in response to MLN4924. (C) Flow cytometry of MCF7 cells showed MLN4924-treated cells were arrested in G1. 
the cell cycle (Figure 5C). Disruption of cell cycle upon MLN4924 is consistent with previous reports in Urothelial carcinoma [32], Ewing Sarcoma [33], Gastric cancer [28], melanoma [34] and breast cancer [35].

\section{DISCUSSION}

It is well established that Mdm2 and $\mathrm{MdmX}$ can form heterodimers via their RING finger domains [9, 1315]. However, how the complex is formed and the possible function(s) of this heterodimer under growth signaling has not been explored. We show that under growth conditions, $\mathrm{MdmX}$ is increased, the MdmX-Mdm2 heterodimer does form, and MdmX is neddylated. This is the first evidence that growth conditions regulate an increase of an oncoprotein complex.

Furthermore, since MdmX levels of all mutants were increased under conditions of growth, signaling through those pathways dependent on the wild-type residues are unlikely to be involved. Although the C464S mutant was tested (Figure 2B), it has been shown that $\mathrm{MdmX}$ overexpression can compensate for ring domain mutants of Mdm2 [9] and one can surmise that the reverse is also true. Since Mdm2 is stabilized when Src is active [23], it is possible that it is able to rescue the C464S mutant of MdmX.

There has been considerable interest in the development of therapeutics that modulate the regulation of kinase pathways and limited development on those that impact other post-translational modifications. One such small molecule, MLN4924, is a specific inhibitor of the NEDD8-activating enzyme in Phase 2 clinical trials. However, its effect on the regulation of p53 has not been fully explored. There have been reports that MLN4924 can elicit DNA stress type responses [35-38] in addition to preventing neddylation.

The majority of studies on the Mdm2-MdmX interaction have been conducted under conditions of
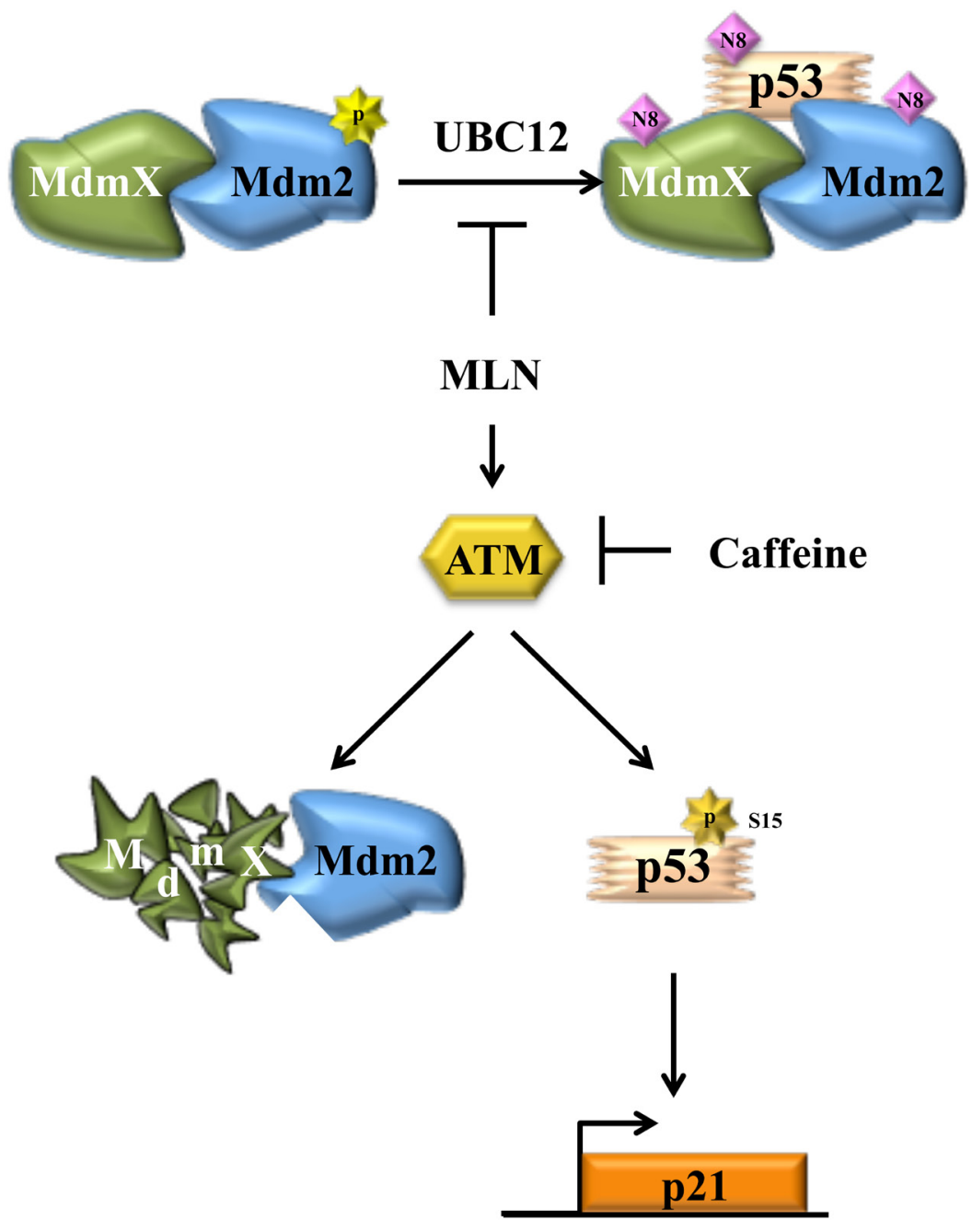

Figure 6: Illustration of how MdmX is integrated into growth factor-mediated neddylation. Under growth conditions, Mdm2 and MdmX form a complex that is necessary to neddylate MdmX and p53. This function is inhibited by MLN4924 which also activates ATM. ATM activation results in conversion of Mdm2 to a ubiquitinating complex, which leads to MdmX destabilization, but not p53 degradation. 
DNA damage. We have shown that c-Abl phosphorylation of Mdm2 results in Mdm2-MdmX complex formation and ubiquitination of $\mathrm{MdmX}$ [16]. Other studies have shown that ATM activation after DNA damage results in ATM-mediated phosphorylation of Mdm2, which blocks nuclear export of p53 [30] and MdmX, which results in rapid destabilization of MdmX. In addition, ATM phosphorylation prevents poly-ubiquitination of p53 by inhibiting homodimerization of $\mathrm{Mdm} 2$ [4]. DNA damage has also been reported to result in checkpoint kinase (Chk) [27, 31, 39, 40] and ribosomal stress pathway [3, 41-44] activation, similarly resulting in $\mathrm{MdmX}$ degradation.

Another component that could be contributing to MdmX degradation under DNA damaging conditions is the activation of NEDP1. This isopeptidase is induced by chemotherapy and can de-neddylate Mdm2 [45]. It is conceivable that the DNA damage response that is initiated by MLN4924 treatment is activating NEDP1, which then de-neddylates $\mathrm{Mdm} 2$ and/or $\mathrm{MdmX}$ resulting in loss of MdmX. Our study illustrates that MdmX stability is sensitive to MLN4924.

The seminal finding of this study is the contribution of MdmX to the neddylation of p53. Initial work suggested the involvement of $\mathrm{MdmX}$ as part of a neddylating complex and reported that while co-expression of $\mathrm{MdmX}$ did not significantly affect Mdm2-mediated neddylation of p53, it was sufficient to rescue Mdm2 mutants that had impaired neddylation activity in transient transfections [9]. However, the mechanism by, and conditions under, which Mdm2 switches from ubiquitinating to neddylating activity was not yet known. In the current study, we show by transient overexpression in $\mathrm{H} 1299$ cells that the maximum amount of neddylated p53 is absolutely dependent on MdmX. Furthermore, if CA-Src is replaced with KD-Src or the cells are treated with MLN4924, this neddylation is diminished. In MEFs, the neddylation pattern is significantly different when MdmX is present compared to when it is absent. Importantly, MdmX contributes to neddylation endogenously as immunoprecipitation of Nedd8 from shMdmX MCF7 cells, followed by western blotting shows much less or almost no neddylated p53 compared to the control. Thus, corroborated by multiple experimental models and approaches, we show that MdmX greatly enhances the neddylation capacity of Mdm2.

Taken together, our results show that during growth conditions when Src is active, Mdm2 and MdmX are in a complex together to neddylate p53 and MdmX. Inhibition of this activity with MLN4924 results in ATM activation, MdmX degradation, and increased p21 (Figure 6). This study highlights the complexity of signaling pathways that affect Mdm2-MdmX enzymatic activity within the cell and emphasizes the need to carefully consider this intricacy in future studies or when targeting this pathway for therapeutic intervention.

\section{MATERIALS AND METHODS}

\section{Cell culture and treatments}

The shMdmX, MdmX, and $\Delta \mathrm{C} \mathrm{MdmX}$ constructs were obtained [46]. Lentiviral CRISPR to Mdm2 was created with forward and reverse Mdm2 CRISPR primers (CACCGTTGGGCCCTTCGTGAGAAT and AAACATTCTCACGAAGGGCCCAAC respectively) to exon 12 cloned into the Lenticrispr vector (pXPR_001 obtained from Clark Wells) as described [47].

Viral transductions were conducted as described [48]. H1299, U87, TMD231, MEF and MCF7 cells were incubated at $37^{\circ} \mathrm{C}$ in a humidified chamber with $5 \% \mathrm{CO}_{2}$ in DMEM high glucose and $8-10 \%$ fetal bovine serum. Transient transfections were performed using 1:1 DNA to PEI (H1299). Equal amounts of DNA were transfected into cells except for neddylation assays (see figure legend). Unless indicated, cells were treated with $0.3 \mu \mathrm{m}$ MLN4924 (Life Sensors), 2 mM Caffeine and/or $10 \mu \mathrm{M}$ ATMi (KU-55933 (Millipore) for $18 \mathrm{~h}$.

\section{Protein analysis}

Cell pellets were lysed in Urea Lysis Buffer (20mM Tris pH6.8, $100 \mathrm{mM} \mathrm{NaH} \mathrm{PO}_{4}, 6 \mathrm{M}$ Urea) on ice for 2 hours and sonicated before centrifugation. After quantitation, proteins were analyzed by western blotting. All antibodies are listed in Supplementary Table 1.

\section{Immunoprecipitation}

Cells were lysed in $25 \mathrm{mM}$ Tris- $\mathrm{HCl}, 150 \mathrm{mM} \mathrm{NaCl}$, $1 \%$ IGEPAL, protease cocktail, $1 \mathrm{mM} \mathrm{Na} \mathrm{VO}_{4}, 10 \mathrm{mM}$ NaF, $1 \mu \mathrm{m}$ Sodium Pyrophosphate $(\mathrm{pH}$ 8.0) and sonicated. Antibodies were preabsorbed, washed and then incubated with lysate at $4^{\circ} \mathrm{C}$ for $2 \mathrm{~h}$. All antibodies are listed in Supplementary Table 1.

\section{Kinase and other in vitro reactions}

Kinase reactions were performed at $30^{\circ} \mathrm{C}$ for 60 minutes in kinase buffer (60 mM HEPES, pH 7.4, $3 \mathrm{mM}$ $\mathrm{MgCl}_{2}, 3 \mathrm{mM} \mathrm{MnCl}, 3 \mu \mathrm{m} \mathrm{Na} \mathrm{VO}_{4}, 20 \mathrm{mM}$ ATP) using $0.1 \mu \mathrm{g}$ of Src (Millipore). Kinase reactions were incubated with glutathione-sepharose beads that were pre-washed in $0.1 \%$ DTT for 30 minutes. This incubation was conducted for 10 minutes in a $0.1 \%$ DTT buffer. Complexes that formed were spun down and washed four times in a high salt buffer (0.5 M KCl, $20 \mathrm{mM}$ Tris, 0.1\% IGEPAL, $\mathrm{pH}$ $8.0)$. The bound proteins were eluted in SDS-Loading Buffer and western blot analysis was performed.

\section{Nickel pull down}

H1299 cells were transfected with His-nedd8 and other plasmids using PEI (1 DNA:1 PEI ratio). Twenty- 
four hours after transfection, cells were lysed in $500 \mu \mathrm{l}$ of $6 \mathrm{M}$ guanidinium- $\mathrm{HCl}, 0.1 \mathrm{M} \mathrm{Na} \mathrm{HPO}_{4} / \mathrm{NaH}_{2} \mathrm{PO}_{4}$, $0.01 \mathrm{M}$ Tris- $\mathrm{HCl} \mathrm{pH} 8.0$ plus $5 \mathrm{mM}$ imidazole and 10 $\mathrm{mM}$ ß-mercaptoethanol. After centrifugation, the lysates were mixed with $30 \mu \mathrm{l}$ of $\mathrm{Ni}^{2+}$-NTA-agarose beads (Qiagen) prewashed with lysis buffer and incubated for $2 \mathrm{~h}$ at room temperature. The beads were successively washed in each of the following: $6 \mathrm{M}$ guanidinium$\mathrm{HCl}, 0.1 \mathrm{M} \mathrm{Na}_{2} \mathrm{HPO}_{4} / \mathrm{NaH}_{2} \mathrm{PO}_{4}, 0.01 \mathrm{M}$ Tris- $\mathrm{HCl} \mathrm{pH}$ 8.0 plus $10 \mathrm{mM}$ ß-mercaptoethanol; $8 \mathrm{M}$ urea, $0.1 \mathrm{M}$ $\mathrm{Na}_{2} \mathrm{HPO}_{4} / \mathrm{NaH}_{2} \mathrm{PO}_{4}, 0.01 \mathrm{M}$ Tris- $\mathrm{HCl} \mathrm{pH} 8.0,10 \mathrm{mM}$ ß-mercaptoethanol; $8 \mathrm{M}$ urea, $0.1 \mathrm{M} \mathrm{Na} 2 \mathrm{HPO}_{4} / \mathrm{NaH}_{2} \mathrm{PO}_{4}$, $0.01 \mathrm{M}$ Tris- $\mathrm{HCl} \mathrm{pH} 6.3,10 \mathrm{mM}$ ß-mercaptoethanol plus $0.2 \%$ Triton X-100; $8 \mathrm{M}$ urea, $0.1 \mathrm{M} \mathrm{Na} \mathrm{HPO}_{4} / \mathrm{NaH}_{2} \mathrm{PO}_{4}$, $0.01 \mathrm{M}$ Tris- $\mathrm{HCl} \mathrm{pH} 6.3,10 \mathrm{mM} \beta$-mercaptoethanol plus $0.1 \%$ Triton X-100. After the last wash the beads were eluted with $200 \mathrm{mM}$ imidazole in 5\% SDS, $0.15 \mathrm{M}$ Tris$\mathrm{HCl} \mathrm{pH} 6.7,30 \%$ glycerol, $0.72 \mathrm{M}$ ß-mercaptoethanol. Eluates were subjected to sodium dodecyl sulfate polyacrylamide gel electrophoresis (SDS-PAGE) and western blotting.

\section{Confocal analysis}

MCF7 cells growing on coverslips were serum starved for 72 hours and then treated for EGF for 8 hours. Cells were washed in PBS and then fixed for one hour at room temperature in $4 \%$ paraformaldehyde. After 3 washes in PBS, cells were permeabilized in $0.1 \%$ Triton-X 100 for 20 minutes. and washed $3 \mathrm{X}$ in PBS. After blocking in 2\% BSA, coverslips were incubated with MdmX and/or Mdm2 antibodies (Red Mdm2 - N20, Green Mdm2 - SMP14, Red MdmX - Bethyl, Green $\mathrm{MdmX}-8 \mathrm{C} 6$ ) overnight at $4^{\circ} \mathrm{C}$ and washed $3 \mathrm{X}$ in PBS. Coverslips were then incubated with AlexaFluor 488 and 637 linked to anti-mouse and anti-rabbit secondary antibodies (respectively) for 1 hour, washed $3 \mathrm{X}$ in PBS, mounted on slides with Prolong Diamond Antifade Mountant with DAPI and visualized with a Leica SP8 MP confocal microscope at room temperature. Images were obtained using HC PL APO 40x/1.3 oil CS2 objective and the Leica Application Suite Advanced Fluorescence Software provided by the Indiana Center for Biological Microscopy core.

\section{Cell cycle analysis}

MCF-7 cells $\left(3 \times 10^{5}\right)$ were plated onto 6-well plates in triplicates and serum starved for $24 \mathrm{~h}$. Cells were released from starvation with DMEM complete and treated with DMSO or MLN $(0.6 \mu \mathrm{M})$ for $18 \mathrm{~h}$, cells were washed with $1 \mathrm{X}$ PBS, harvested with trypsin, and spun at $300 \mathrm{~g}$ x 5 minutes. FACS analysis was performed on the Muse (Millipore) with the Muse Cell Cycle Kit, according to manufacturers instructions (5,000 events). Significance was calculated using Two-tailed Student's T-Test.

\section{Abbreviations}

Mdm2: Mouse double minute 2; MEFs: mouse embryonic fibroblasts; ATM: Ataxia Telangiectasia Mutated; RING: Really Interesting New Gene; EGF: epidermal growth factor; c-Abl: Abelson murine leukemia viral oncogene homolog 1; DMSO: Dimethyl sulfoxide; CRISPR: Clustered regularly interspaced short palindromic repeats; chk: checkpoint kinase; Nedd8: Neural Precursor Cell Expressed, Developmentally DownRegulated 8; NEDP1: NEDD8-Specific Protease 1; CA: constitutively active; KD: kinase dead; C: control; WB: western blot; sh: short hairpin; HA: Human influenza hemagglutinin aa 98-106; Caffeine: Caff.

\section{CONFLICTS OF INTEREST}

The authors declare that they have no conflicts of interest.

\section{FUNDING}

This work was supported by the National Institutes of Health Grant CA172256 (to L.D.M).

\section{REFERENCES}

1. Onel K, Cordon-Cardo C. MDM2 and prognosis. Mol Cancer Res. 2004; 2:1-8.

2. Rayburn E, Zhang R, He J, Wang H. MDM2 and human malignancies: expression, clinical pathology, prognostic markers, and implications for chemotherapy. Curr Cancer Drug Targets. 2005; 5:27-41.

3. Cheng Q, Chen J. Mechanism of p53 stabilization by ATM after DNA damage. Cell Cycle. 2010; 9:472-8.

4. Cheng Q, Chen L, Li Z, Lane WS, Chen J. ATM activates p53 by regulating MDM2 oligomerization and E3 processivity. EMBO J. 2009; 28:3857-67. https://doi. org/10.1038/emboj.2009.294.

5. Dolezelova P, Cetkovska K, Vousden KH, Uldrijan S. Mutational analysis of Mdm2 C-terminal tail suggests an evolutionarily conserved role of its length in Mdm2 activity toward p53 and indicates structural differences between Mdm2 homodimers and Mdm2/MdmX heterodimers. Cell Cycle. 2012; 11:953-62. https://doi.org/10.4161/ cc.11.5.19445.

6. Kawai H, Lopez-Pajares V, Kim MM, Wiederschain D, Yuan ZM. RING domain-mediated interaction is a requirement for MDM2's E3 ligase activity. Cancer Res. 2007; 67:6026-30. https://doi.org/10.1158/0008-5472. CAN-07-1313.

7. Leslie PL, Ke H, Zhang Y. The MDM2 RING Domain and Central Acidic Domain Play Distinct Roles in MDM2 Protein Homodimerization and MDM2-MDMX Protein 
Heterodimerization. J Biol Chem. 2015; 290:12941-50. https://doi.org/10.1074/jbc.M115.644435.

8. Poyurovsky MV, Priest C, Kentsis A, Borden KL, Pan ZQ, Pavletich N, Prives C. The Mdm2 RING domain $\mathrm{C}$-terminus is required for supramolecular assembly and ubiquitin ligase activity. EMBO J. 2007; 26:90-101. https:// doi.org/10.1038/sj.emboj.7601465.

9. Singh RK, Iyappan S, Scheffner M. Hetero-oligomerization with $\mathrm{MdmX}$ rescues the ubiquitin/Nedd8 ligase activity of RING finger mutants of Mdm2. J Biol Chem. 2007; 282:10901-7. https://doi.org/10.1074/jbc.M610879200.

10. Uldrijan S, Pannekoek WJ, Vousden KH. An essential function of the extreme C-terminus of MDM2 can be provided by MDMX. EMBO J. 2007; 26:102-12. https:// doi.org/10.1038/sj.emboj.7601469.

11. Badciong JC, Haas AL. MdmX is a RING finger ubiquitin ligase capable of synergistically enhancing Mdm2 ubiquitination. J Biol Chem. 2002; 277:49668-75. https:// doi.org/10.1074/jbc.M208593200.

12. Huang L, Yan Z, Liao X, Li Y, Yang J, Wang ZG, Zuo Y, Kawai H, Shadfan M, Ganapathy S, Yuan ZM. The p53 inhibitors MDM2/MDMX complex is required for control of p53 activity in vivo. Proc Natl Acad Sci U S A. 2011; 108:12001-6. https://doi.org/10.1073/pnas.1102309108.

13. Linke K, Mace PD, Smith CA, Vaux DL, Silke J, Day CL. Structure of the MDM2/MDMX RING domain heterodimer reveals dimerization is required for their ubiquitylation in trans. Cell Death Differ. 2008; 15:841-8. https://doi. org/10.1038/sj.cdd.4402309.

14. Tanimura S, Ohtsuka S, Mitsui K, Shirouzu K, Yoshimura A, Ohtsubo M. MDM2 interacts with MDMX through their RING finger domains. FEBS Lett. 1999; 447:5-9.

15. Wang $X$, Jiang $X . M d m 2$ and $M d m X$ partner to regulate p53. FEBS Lett. 2012; 586:1390-6. https://doi. org/10.1016/j.febslet.2012.02.049.

16. Waning DL, Lehman JA, Batuello CN, Mayo LD. c-Abl phosphorylation of $\mathrm{Mdm} 2$ facilitates Mdm2-Mdmx complex formation. J Biol Chem. 2011; 286:216-22. https:// doi.org/10.1074/jbc.M110.183012.

17. Waning DL, Lehman JA, Batuello CN, Mayo LD. Controlling the Mdm2-Mdmx-p53 Circuit. Pharmaceuticals (Basel). 2010; 3:1576-93. https://doi.org/10.3390/ ph3051576.

18. Shvarts A, Steegenga WT, Riteco N, van Laar T, Dekker P, Bazuine M, van Ham RC, van der Houven van Oordt W, Hateboer G, van der Eb AJ, Jochemsen AG. MDMX: a novel p53-binding protein with some functional properties of MDM2. EMBO J. 1996; 15:5349-57.

19. Yu Q, Li Y, Mu K, Li Z, Meng Q, Wu X, Wang Y, Li L. Amplification of Mdmx and overexpression of MDM2 contribute to mammary carcinogenesis by substituting for p53 mutations. Diagn Pathol. 2014; 9:71. https://doi. org/10.1186/1746-1596-9-71.
20. Laurie NA, Donovan SL, Shih CS, Zhang J, Mills N, Fuller C, Teunisse A, Lam S, Ramos Y, Mohan A, Johnson D, Wilson M, Rodriguez-Galindo C, et al. Inactivation of the p53 pathway in retinoblastoma. Nature. 2006; 444:61-6. https://doi.org/10.1038/nature05194.

21. Gembarska A, Luciani F, Fedele C, Russell EA, Dewaele M, Villar S, Zwolinska A, Haupt S, de Lange J, Yip D, Goydos J, Haigh JJ, Haupt Y, et al. MDM4 is a key therapeutic target in cutaneous melanoma. Nat Med. 2012; 18:1239-47. https://doi.org/10.1038/nm.2863.

22. Xirodimas DP, Saville MK, Bourdon JC, Hay RT, Lane DP. Mdm2-mediated NEDD8 conjugation of p53 inhibits its transcriptional activity. Cell. 2004; 118:83-97. https://doi. org/10.1016/j.cell.2004.06.016.

23. Batuello CN, Hauck PM, Gendron JM, Lehman JA, Mayo LD. Src phosphorylation converts Mdm2 from a ubiquitinating to a neddylating E3 ligase. Proc Natl Acad Sci U S A. 2015; 112:1749-54. https://doi.org/10.1073/ pnas. 1416656112 .

24. Linares LK, Hengstermann A, Ciechanover A, Muller S, Scheffner M. HdmX stimulates Hdm2-mediated ubiquitination and degradation of p53. Proceedings of the National Academy of Sciences of the United States of America. 2003; 100:12009-14. https://doi.org/10.1073/ pnas.2030930100.

25. Pan Y, Chen J. MDM2 promotes ubiquitination and degradation of MDMX. Mol Cell Biol. 2003; 23:5113-21.

26. Zuckerman V, Lenos K, Popowicz GM, Silberman I, Grossman T, Marine JC, Holak TA, Jochemsen AG, Haupt Y. c-Abl phosphorylates Hdmx and regulates its interaction with p53. J Biol Chem. 2009; 284:4031-9. https://doi. org/10.1074/jbc.M809211200.

27. Jin Y, Dai MS, Lu SZ, Xu Y, Luo Z, Zhao Y, Lu H. 14-3-3gamma binds to MDMX that is phosphorylated by UV-activated Chk1, resulting in p53 activation. EMBO J. 2006; 25:1207-18. https://doi.org/10.1038/ sj.emboj.7601010.

28. Lan H, Tang Z, Jin H, Sun Y. Neddylation inhibitor MLN4924 suppresses growth and migration of human gastric cancer cells. Sci Rep. 2016; 6:24218. https://doi. org/10.1038/srep24218.

29. Cheng Q, Cross B, Li B, Chen L, Li Z, Chen J. Regulation of MDM2 E3 ligase activity by phosphorylation after DNA damage. Mol Cell Biol. 2011; 31:4951-63. https://doi. org/10.1128/MCB.05553-11.

30. Maya R, Balass M, Kim ST, Shkedy D, Leal JF, Shifman O, Moas M, Buschmann T, Ronai Z, Shiloh Y, Kastan MB, Katzir E, Oren M. ATM-dependent phosphorylation of Mdm2 on serine 395: role in p53 activation by DNA damage. Genes Dev. 2001; 15:1067-77. https://doi. org/10.1101/gad.886901.

31. Chen L, Gilkes DM, Pan Y, Lane WS, Chen J. ATM and Chk2-dependent phosphorylation of MDMX contribute to 
p53 activation after DNA damage. EMBO J. 2005; 24:341122. https://doi.org/10.1038/sj.emboj.7600812.

32. Kuo KL, Ho IL, Shi CS, Wu JT, Lin WC, Tsai YC, Chang HC, Chou CT, Hsu CH, Hsieh JT, Chang SC, Pu YS, Huang KH. MLN4924, a novel protein neddylation inhibitor, suppresses proliferation and migration of human urothelial carcinoma: in vitro and in vivo studies. Cancer Lett. 2015; 363:127-36. https://doi.org/10.1016/j.canlet.2015.01.015.

33. Mackintosh C, Garcia-Dominguez DJ, Ordonez JL, GinelPicardo A, Smith PG, Sacristan MP, de Alava E. WEE1 accumulation and deregulation of S-phase proteins mediate MLN4924 potent inhibitory effect on Ewing sarcoma cells. Oncogene. 2013; 32:1441-51. https://doi.org/10.1038/ onc. 2012.153

34. Blank JL, Liu XJ, Cosmopoulos K, Bouck DC, Garcia K, Bernard H, Tayber O, Hather G, Liu R, Narayanan U, Milhollen MA, Lightcap ES. Novel DNA damage checkpoints mediating cell death induced by the NEDD8activating enzyme inhibitor MLN4924. Cancer Res. 2013; 73:225-34. https://doi.org/10.1158/0008-5472. CAN-12-1729.

35. Bailly A, Perrin A, Bou Malhab LJ, Pion E, Larance M, Nagala M, Smith P, O'Donohue MF, Gleizes PE, Zomerdijk J, Lamond AI, Xirodimas DP. The NEDD8 inhibitor MLN4924 increases the size of the nucleolus and activates p53 through the ribosomal-Mdm2 pathway. Oncogene. 2016; 35:415-26. https://doi.org/10.1038/onc.2015.104.

36. Ho IL, Kuo KL, Liu SH, Chang HC, Hsieh JT, Wu JT, Chiang CK, Lin WC, Tsai YC, Chou CT, Hsu CH, Pu YS, Shi CS, et al. MLN4924 Synergistically Enhances Cisplatin-induced Cytotoxicity via JNK and Bcl-xL Pathways in Human Urothelial Carcinoma. Sci Rep. 2015; 5:16948. https://doi.org/10.1038/srep16948.

37. Jia L, Li H, Sun Y. Induction of p21-dependent senescence by an NAE inhibitor, MLN4924, as a mechanism of growth suppression. Neoplasia. 2011; 13:561-9.

38. Lin JJ, Milhollen MA, Smith PG, Narayanan U, Dutta A. NEDD8-targeting drug MLN4924 elicits DNA rereplication by stabilizing Cdt1 in $\mathrm{S}$ phase, triggering checkpoint activation, apoptosis, and senescence in cancer cells. Cancer Res. 2010; 70:10310-20. https://doi.org/10.1158/0008-5472. CAN-10-2062.
39. LeBron C, Chen L, Gilkes DM, Chen J. Regulation of MDMX nuclear import and degradation by Chk2 and 14-33. EMBO J. 2006; 25:1196-206. https://doi.org/10.1038/ sj.emboj.7601032.

40. Wu S, Chen L, Becker A, Schonbrunn E, Chen J. Casein kinase 1alpha regulates an MDMX intramolecular interaction to stimulate p53 binding. Mol Cell Biol. 2012; 32:4821-32. https://doi.org/10.1128/MCB.00851-12.

41. Chen J. The Roles of MDM2 and MDMX Phosphorylation in Stress Signaling to p53. Genes Cancer. 2012; 3:274-82. https://doi.org/10.1177/1947601912454733.

42. Daftuar L, Zhu Y, Jacq X, Prives C. Ribosomal proteins RPL37, RPS15 and RPS20 regulate the Mdm2-p53MdmX network. PLoS One. 2013; 8:e68667. https://doi. org/10.1371/journal.pone.0068667.

43. Gilkes DM, Chen J. Distinct roles of MDMX in the regulation of $\mathrm{p} 53$ response to ribosomal stress. Cell Cycle. 2007; 6:151-5. https://doi.org/10.4161/cc.6.2.3719.

44. Gilkes DM, Chen L, Chen J. MDMX regulation of p53 response to ribosomal stress. EMBO J. 2006; 25:5614-25. https://doi.org/10.1038/sj.emboj.7601424.

45. Watson IR, Li BK, Roche O, Blanch A, Ohh M, Irwin MS. Chemotherapy induces NEDP1-mediated destabilization of MDM2. Oncogene. 2010; 29:297-304. https://doi. org/10.1038/onc.2009.314.

46. Patton JT, Mayo LD, Singhi AD, Gudkov AV, Stark GR, Jackson MW. Levels of HdmX expression dictate the sensitivity of normal and transformed cells to Nutlin-3. Cancer Res. 2006; 66:3169-76. https://doi. org/10.1158/0008-5472.CAN-05-3832.

47. Shalem O, Sanjana NE, Hartenian E, Shi X, Scott DA, Mikkelsen TS, Heckl D, Ebert BL, Root DE, Doench JG, Zhang F. Genome-scale CRISPR-Cas9 knockout screening in human cells. Science. 2014; 343:84-7. https://doi. org/10.1126/science.1247005.

48. Jackson MW, Patt LE, LaRusch GA, Donner DB, Stark GR, Mayo LD. Hdm2 nuclear export, regulated by insulinlike growth factor-I/MAPK/p90Rsk signaling, mediates the transformation of human cells. J Biol Chem. 2006; 281:16814-20. https://doi.org/10.1074/jbc.M511617200. 